\title{
THE NEED FOR PHYSICAL MODELS IN COASTAL ENGINEERING
}

\author{
Edward Couriel, Manly Hydraulics Laboratory, edward.couriel@mhl.nsw.gov.au \\ Lex Nielsen, Advisian, lex.nielsen@advisian.com \\ Indra Jayewardene, Manly Hydraulics Laboratory, Indra.Jayewardene@mhl.nsw.gov.au \\ Bronson McPherson, Manly Hydraulics Laboratory, bronson.mcpherson@mhl.nsw.gov.au
}

\section{INTRODUCTION}

Coastal process understanding is based on observations of physical processes in the field and laboratory from which theories are developed. Theories may be expressed mathematically and coded into numerical models. Compared with the cost of field data acquisition and laboratory experiments, the cost of numerical modelling is often perceived to be low. Therefore, there is a tendency to undertake coastal investigation and design using numerical modelling methods alone. However, there is still a need for physical modelling in coastal engineering.

NUMERICAL MODEL APPPLICATION \& LIMITATIONS Numerical models may be empirical or analytical. Empirical formulations comprise the identification of relevant parameters relating cause and effect and the development of relationships between these parameters using coefficients derived from many field or laboratory observations (e.g., Hudson (1953) armour size formula, O'Brien (1931) tidal prism/inlet area relationship) whereas analytical approaches comprise the development and application of formulae derived from mechanism understanding (principally based on the conservation of mass, momentum and energy).

Once developed, the numerical model represents the understanding of the theory at the time of its development. The models are most useful when validated for the interpolation of data between validation points, such as wave transformation studies and assessing the impacts of domain perturbations such as port dredging.

However, numerical modelling has limitations including:

- Spatial (1D, 2D or 3D) and temporal (steady state, unsteady time-averaged and dynamic) simplifications (Toombes and Chanson, 2011).

- Primarily, a numerical model is locked into past thinking and cannot discover new processes. Kamphuis (2016) stated that numerical models do not add to the understanding of the problem but, simply, reflect the input by the model's creators.

- Rarely is it possible to validate exactly a numerical model simultaneously at a spread of locations over the domain, which limits the accuracy and the validity of data interpolation over the model domain.

- The random nature of natural processes is not conducive to parameterization, inducing large spreads in validation data and uncertainty in results.

PHYSICAL MODEL APPPLICATION \& LIMITATIONS Hughes (2014) showed that numerical models have not made laboratory-based research obsolete and defined the critical roles of laboratory research and physical modeling as:

(1) confirming coastal designs

(2) developing empirical design guidance

(3) contributing to numerical model development by elucidating physical processes

(4) verifying numerical models.

For example, in respect of (1), every breakwater or revetment is different and has not been tested in a flume, so the Hudson (1931) approach cannot be relied upon except for concept design.

Further to points (2) and (3), because physical models behave in a manner that is similar to the prototype and they provide a direct visual impression of the processes, physical models add to the understanding of the problem (Kamphuis, 2016). This is a fundamental difference from numerical models.

In respect of (4), Hughes (2014) found that about half of the papers cited used laboratory data to support development and validation of numerical models and that this had not changed much over 20 years.

A further role of physical modelling is that lab experimentation and simulation experience are excellent demonstration tools (Kamphuis, 2016) where highly variable natural inputs can be controlled.

However, limitations of physical models include:

- Impossibility to attain complete similarity (matching ratios of predominant forces in model and prototype)

- Similitude by one law (Froude, Reynolds, Weber or Mach scaling) violates others (scale effects)

Good knowledge of established physical laws, judgement and experience are vital in selecting the appropriate model methods. In making the necessary compromises selecting scales and test conditions, modelling becomes an art as well as a science.

\section{MODELLING CASE 1 - LITTORAL DRIFT TRANSPORT}

Based on the difficulties regarding similitude, researchers have proffered variously on effective axioms for modelling suspension-dominated processes. The challenge is to make an informed decision as to the most appropriate scaling formulation for a situation.

On an eroding beach in Botany Bay, NSW, Australia, works comprising submerged groynes to stabilise the beach were designed using a mobile bed physical model at NSW Government's Manly Hydraulics Laboratory (MHL) using lightweight sediments as numerical modelling was not doable. Completed works in Figure 1 compare well the model with prototype beach planforms. 


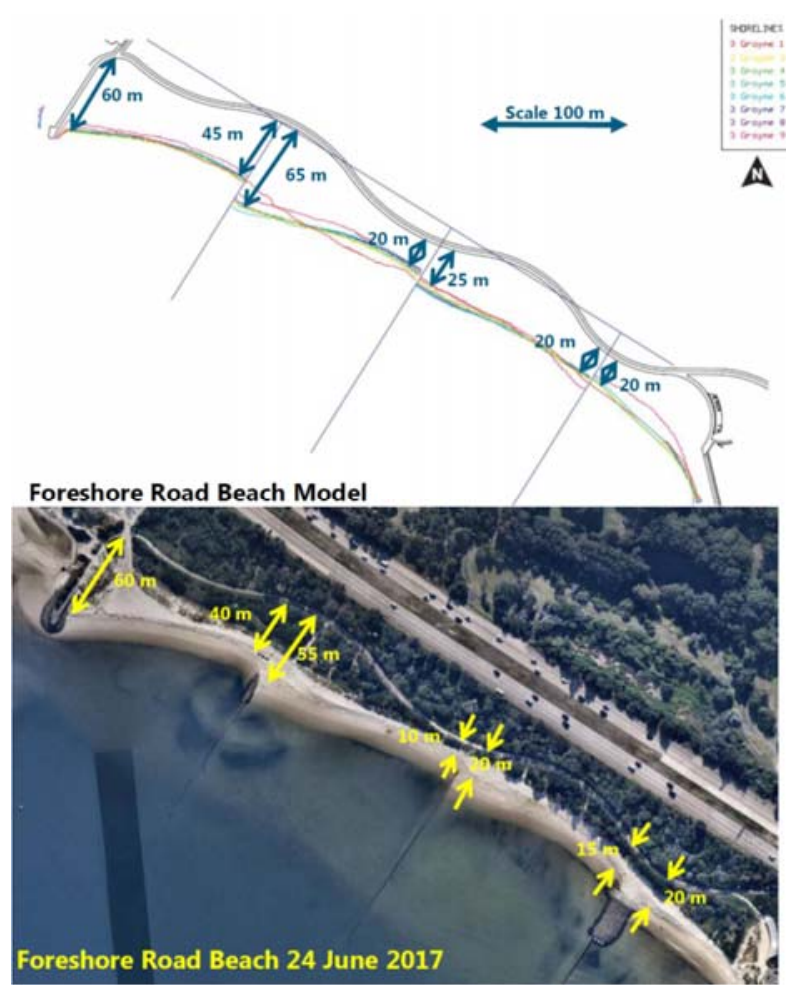

Figure 1 - Model verses prototype shorelines

Although violating every axiom for scale modelling of sediment transport, the physical model proved to be a cost-effective tool for predicting medium-term wavegenerated planform beach responses to nearshore structures. The salient precepts of the small-scale basin modelling that enabled its application to determine foreshore planform were the representative nearshore wave energy gradients in the model and an ability for the model waves to readily mobilise the sediments.

\section{MODELLING CASE 2 - HARBOUR SEICHING}

A public boat ramp in Coffs Harbour, NSW, Australia has suffered from dangerous long-wave effects for many years. Numerical modelling using Boussinesq (BW) waves forced by white noise carried out in a previous investigation predicted reductions up to $50 \%$ due to the proposed change of planform of the boat ramp harbour. Detailed design was undertaken using MHL's wave basin with measured short wave spectra incorporating group bounded long waves and surf beat as input to create the measured long-wave responses in the wider harbour and local boat ramp harbour seiche before testing the proposed boat ramp harbour alterations.

The physical model results indicated not more than $23 \%-29 \%$ reduction in the long-wave amplitudes following further planform optimisation in the physical model. Field measurements of the long-wave response following construction were consistent with the expectations created during detailed design using the physical model. While great advances have been made in unsteady dynamic numerical wave models that allow long-wave responses to be effectively represented for simple geometries, the complex multi-basin interactions of Coffs Harbour were demonstrated to be far better represented in a physical model.

MODELLING CASE 3 - SUBMERGED ROCK ARMOUR Both $2 D$ and $3 D$ wave models were used to assess the stability of rock rubble mound protection for a submerged gas pipeline, including details for the shore crossing.

Preliminary testing demonstrated that the analytically based design would have failed leading to design amendments. The subsequent 3D modelling demonstrated further unacceptable failures which led to a rock berm design to mitigate focusing attributable to adjacent bathymetry that resulted in significant rock armour size savings and prevented failure that would have resulted from the original analytically based design.

\section{CONCLUSIONS}

Good knowledge of relevant physical laws and boundary conditions, judgement and experience are all demonstrated to be vital in selecting appropriate methods for both numerical and physical models. In making the compromises needed, effective modelling is an art of experience as much as science. When undertaken correctly, physical models help all observers (irrespective of background) better understand the crux of the matter, often amid great complexity.

Irrespective of the modelling approach undertaken, a thorough understanding of the key physical processes operating is vital, and this can be enhanced only by way of prototype validation of model assumptions and outputs following commissioning of works, especially the documentation of model short-comings.

\section{REFERENCES}

Hudson, R.Y. (1953). Wave Forces on Breakwaters, Transactions of the American Society of Civil Engineers, ASCE, Vol. 118, 1953, pp653-674.

Hughes, S.A. (2014). Coastal engineering challenges in a changing world, Journal of Applied Water Engineering and Research, 2014, Vol. 2, No. 2, pp72-80.

Kamphuis, J.W. (1985). On understanding scale effect in coastal mobile bed models, in Dalrymple, R.A. (Ed.) Physical Modelling in Coastal Engineering, Balkema, 141-162.

Kamphuis, J.W. (2016). Coastal modeling - a retrospective, Proc. 6th International Conference on the Application of Physical Modelling in Coastal and Port Engineering and Science (Coastlab16), Ottawa, Canada, May 10-13, 2016.

O'Brien, M.P. (1931). Estuary tidal prism related to entrance areas, Civil Engineering, Vol. 1, No. 8, p 738.

Toombes, L., Chanson (2011). Numerical limitations of hydraulic models. $34^{\text {th }}$ IAHR World Congress, Brisbane, pp2322-2329. 